\title{
Impact of Individualized Hemolysis Management Based on Biological Variation Cut-offs in a Clinical Laboratory
}

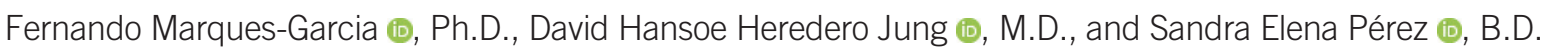 \\ Department of Clinical Biochemistry, University Hospital of Salamanca, Salamanca, Spain
}

Background: Hemolysis is the most common type of preanalytical interference. Cut-offs based on the hemolysis index level can be established using different approaches. The Working Group for Preanalytical Phase of the European Federation of Laboratory Medicine has developed a protocol for hemolysis management based on cut-offs estimated from biological variation (BV) and the use of interpretative comments. We developed and assessed the implementation of the protocol in our laboratory.

Methods: Hemolysates from whole blood were prepared following the Meites method, and pooled serum samples with known $\mathrm{Hb}$ concentrations were prepared. For each analyte (42 ), interferograms were generated and used to establish cut-offs: desirable analytical quality specification and reference change value. This protocol was assessed, both preand post-implementation, according to expert rules in the Laboratory Information System.

Results: Among the analytes evaluated, we selected those that showed the highest degree of hemolysis interference: lactate dehydrogenase (LDH), aspartate aminotransferase, direct bilirubin, potassium, and folic acid. The cut-offs for LDH and direct bilirubin were the lowest. Only $28.16 \%$ of all LDH values were adequately reported in the pre-implantation retrospective study, but this percentage improved in the post-implementation stage.

Conclusions: The development and implementation of a harmonized protocol for hemolysis management based on BV cut-offs and result reporting significantly improve hemolysis detection and lead to a decrease in the number of hemolyzed samples over time.

Key Words: Hemolysis, Interference, Biological variation, Cut-off, Harmonization
Received: February 7, 2021

Revision received: June 11,2021

Accepted: September 16, 2021

\section{Corresponding author:}

Fernando Marques-Garcia, Ph.D. Department of Clinical Biochemistry, University Hospital of Salamanca,

Salamanca, Spain

Tel: +34-923-291100

E-mail: f.marg@hotmail.es

\section{INTRODUCTION}

A significant number of errors occur during the total testing process in the preanalytical phase in clinical laboratories, accounting for $40 \%-70 \%$ of total errors [1-4]. Hemolysis is the most common type of preanalytical interference; it occurs more frequently than high bilirubin and lipemia, and leads to errors in result interpretation, with potential damaging consequences for the patient. Therefore, adequate hemolysis management has a great impact on patient health [5-7]. Hemolysis is defined as the rupture of red blood cells (RBC) and the release of their contents (as well as white blood cell and platelet contents). It can occur in vivo, e.g., in hemolytic anemias of acquired and/or genetic origin, or in vitro during sample collection, transport, and storage [6, 8]. Cell rupture and content release into the plasma cause interferences in the determination of blood analytes via various mechanisms: an increase in the concentration of the released constituent, chemical interference, spectrophotometric interference, or a dilutional effect [6].

Visual assessment of the degree of hemolysis after sample 
centrifugation has been historically used to evaluate interference; however, this method is no longer recommended. Currently, the use of automated analyzers to determine the hemolysis index $(\mathrm{HI})$ is recommended, as it is a standardized and more accurate method than visual assessment [5, 9]. According to the manufacturer, the $\mathrm{HI}$ may be presented as a scale without units or as concentration units. Currently, only two manufacturers provide biochemical tests (Roche Diagnostics, Basel, Switzerland and Abbott, Abbott Park, IL, USA), and one manufacturer provides a coagulation test (ACLTop-Werfen, Barcelona, Spain), in which the hemolysis level may be converted to a free $\mathrm{Hb}$ concentration (measured in $\mathrm{g} / \mathrm{L}$ ) $[10,11]$. However, there exist discrepancies in results across laboratories and a lack of unified criteria for hemolysis management. A questionnaire survey among 846 laboratory professionals in the United States revealed that only 40\%$46 \%$ had standardized hemolysis reports for lactate dehydrogenase (LDH), potassium, and glucose [8]. To determine the degree of hemolysis, an interference cut-off has been established as a maximum bias of $\pm 10 \%$ from a non-hemolyzed baseline pool [10]. In contrast, the CLSI-C56-A guidelines report acceptability criteria based on the biological variation (BV) and reference change value (RCV) for each analyte [12]. To standardize this procedure, the Working Group for Preanalytical Phase (WGPRE) of the European Federation of Clinical Chemistry and Laboratory Medicine (EFLM) has developed a protocol for the determination of cut-offs and their inclusion in laboratory report comments [13].

We report the implementation and analyte-by-analyte evaluation of a protocol for the management of hemolyzed samples based on the protocol presented by the EFLM WG-PRE. Additionally, we describe our experience in assessing the impact on the management of interference after the protocol implementation.

\section{MATERIALS AND METHODS}

\section{Samples}

Residual serum samples from routine analyses were collected in tubes without anticoagulant and with separating gel (Vacuette; Greiner Bio-One, Madrid, Spain). The samples were stored at $4^{\circ} \mathrm{C}$ and analyzed within 24 hours. Forty-two biochemical analytes were measured: 31 in the Cobas c701 module and 11 in the Cobas e602 module (Roche Diagnostics). The study was conducted using equipment under stable conditions (over six months with stable internal quality control), and equipment performance was assessed via participation in an external quality assurance program of the Spanish Society of Laboratory Medicine (SEQCML). The internal control materials used were Liquid Assayed Multiqual Levels 1 and 2, and Liquicheck Immunoassay Plus Levels 1 and 2 (Bio-Rad Laboratories, Hercules, CA, USA). Control data were obtained from the Laboratory Information System (LIS), Cobas Infinity IT Solutions (Roche Diagnostics). The study protocol was approved by the ethical review board of Salamanca University Hospital, Salamanca, Spain and was in agreement with the World Medical Association Declaration of Helsinki (2017).

\section{Preparation of hemolysates and serum pool}

Hemolysates were prepared by RBC lysis through osmotic disruption within 12 hrs after sample collection, according to the Meites method [14]. Five milliliters of total blood in Vacuette Lithium Heparin tubes was centrifuged at $1,500 \times$ g for 5 minutes. The supernatant was discarded, and the RBC were washed three times with a $0.9 \%$ sodium chloride solution. Next, distilled water was added in a 1:1 ratio with RBC and the mixture was stored at $-20^{\circ} \mathrm{C}$ overnight. The next day, the tubes were thawed and centrifuged at $1,500 \times g$ for 5 minutes. The $\mathrm{Hb}$ concentration in the supernatant was measured using an hematology analyzer, SysmexXN-2000 (Roche Diagnostics). The hemolysates were stored at $-20^{\circ} \mathrm{C}$ in $500 \mu \mathrm{L}$ aliquots.

On the day of the interference study, a fresh pool was prepared by mixing six serum samples in which the levels of hemolysis, icterus, lipemia, and the analytes evaluated in this study were within the reference intervals established in the laboratory. Appropriate hemolysate volumes were added to obtain $\mathrm{Hb}$ concentrations of $0,0.25,0.5,1,2,3,5$, and $10 \mathrm{~g} / \mathrm{L}$. Aliquots were prepared in duplicate.

The degree of hemolysis is indicated by the $\mathrm{HI}$, which gives a semiquantitative estimation of the free $\mathrm{Hb}$ concentration in the sample through several dichromatic absorbance measurements at $600 / 570 \mathrm{~nm}$ or $415 \mathrm{~nm}$. The deviation percentage for each analyte caused by hemolysis or bias was determined using the following formula: bias $=\left(T_{1}-T_{0} / T_{0}\right) \times 100$, where $T_{0}$ is the mean concentration of the analyte in samples without hemolysis and $T_{1}$ is the mean concentration of the analyte measured in the pool with added hemolysate. The experiment was repeated three times in duplicate.

\section{Interferograms}

Interference was assessed according to the method reported by Glick, et al. [15], presenting the results as relative percentages of deviation of the concentration of each analyte from the initial 


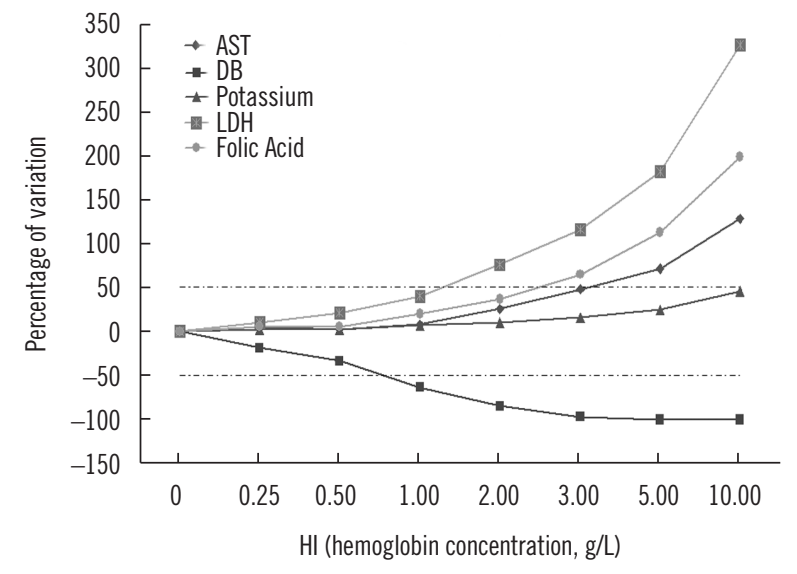

\begin{tabular}{lrrrrrrrr}
\hline & \multicolumn{8}{c}{ HI (Hb concentration) $(\mathrm{g} / \mathrm{L})$} \\
\cline { 2 - 9 } Analyte & 0 & 0.25 & 0.50 & \multicolumn{1}{c}{1} & \multicolumn{1}{c}{2} & \multicolumn{1}{c}{3} & \multicolumn{1}{c}{5} & \multicolumn{1}{c}{10} \\
\hline AST & 0 & 5.70 & 2.90 & 8.6 & 25.7 & 48.6 & 71.4 & 128.6 \\
DB & 0 & -18.20 & -33.30 & -63.6 & -84.8 & -97.0 & -100.0 & -100.0 \\
Potassium & 0 & 2.40 & 2.40 & 7.2 & 10.8 & 15.7 & 25.3 & 45.8 \\
LDH & 0 & 10.60 & 21.20 & 41.0 & 76.4 & 116.6 & 182.2 & 325.3 \\
Folic acid & 0 & 6.20 & 6.00 & 20.8 & 37.5 & 64.5 & 112.3 & 198.2 \\
\hline
\end{tabular}

Fig. 1. LDH and DB were the most significantly influenced by the presence of hemolysis in a sample. Representation of the model proposed by Glick, et al. [15] for the five selected analytes. It compares the percentage of variation in analyte concentration according to the free $\mathrm{Hb}$ concentration in the sample. The dotted lines indicate the interference level of $50 \%$.

Abbreviations: AST, aspartate aminotransferase; DB, direct bilirubin; LDH, lactate dehydrogenase; $\mathrm{HI}$, hemolysis index.

concentration. The data were represented graphically with the $\mathrm{HI}$ on the $\mathrm{X}$-axis and the deviation percentage of each analyte concentration on the Y-axis. We used the interferogram dates to calculate the straight-line trend value and $\mathrm{R}^{2}$ regression coefficient. In all cases, the selected regression model showed $R^{2}>0.98$ [16]. Interferograms for the five selected analytes were generated using the data described in Fig. 1.

\section{RCV determination}

The RCV was calculated according to the following formula [16]:

$$
R C V=\text { square } \operatorname{root}(2) \times 1.96 \times\left(\text { square } \operatorname{root}\left(\left(C V_{I}\right)^{2}+\left(C V_{A}\right)^{2}\right)\right) \text {, }
$$

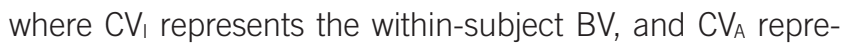
sents the analytical variation in a given laboratory for a given method. $C V_{A}$ was obtained from data collected from an internal quality control process of the laboratory over a period of six months prior to the beginning of the study, using the following formula:

$$
\mathrm{CV}_{\mathrm{A}}=(\mathrm{SD} / \mathrm{mean}) \times 100
$$

\section{Cut-off selection}

Out of all analytes, five were selected: aspartate aminotransferase (AST), direct bilirubin (DB), potassium, LDH, and folic acid. The selection criterion was a percentage of hemolysis interference $>50 \%$ for each analyte with a $\mathrm{Hb}$ concentration of $5 \mathrm{~g} / \mathrm{L}$ (Fig. 1). For the five analytes selected, two cut-offs were established based on analytical criteria and the variation in concentration between two consecutive points (RCV) [13]. As an analytical acceptance limit, based on the BV, we established the desirable quality specification (DQS) for systematic error (desirable systematic error [SE]) as a cut-off. This value was obtained from the SEQC ${ }^{\mathrm{ML}} \mathrm{BV}$ database [18]. For each analyte, the data were compared with those published in the EFLM BV database [19]. The second cut-off was the RCV [13]. By including analytical variation and $\mathrm{BV}$, an interference level above this value may represent a high impact on the therapeutic choice made (clinical cut-off). To establish hemolysis values for both cut-offs, the model used regression lines, calculated based on the interferograms described above.

\section{Expert rule development}

Starting from the established cut-offs, expert rules were formulated to report the presence of hemolysis in samples. In accordance with the harmonization document of the EFLM WG-PRE [13], ranges were established based on the cut-offs. These ranges were then used to determine whether the result should be reported with or without an informative comment, depending on the hemolysis degree detected. Four intervals were established for each analyte: (1) below the desirable SE: the result is reported without comments; (2) between the desirable SE and RCV: the result is reported, and a comment is added to mention the effect of the interference (underestimation or overestimation); (3) above the RCV: the result is not reported, and an informative comment is added to mention the reason for the rejection; (4) $\mathrm{HI}>10 \mathrm{~g} / \mathrm{L}$ : the results of the entire request are canceled. Once these rules were established, they were implemented and automated in the LIS Cobas Infinity IT Solutions.

\section{Pre-implementation retrospective study}

To assess the impact of the implementation of a hemolysis interference information system based on the above cut-offs, we conducted a retrospective analysis of hemolysis samples collected between December 2017 and April 2018 (five months) in both the emergency laboratory and the routine biochemistry laboratory. Folic acid concentrations were analyzed only in routine samples. Frequency distributions (in percentage) of the re- 
sults of the HI for AST, DB, potassium, LDH, and folic acid were determined according to the intervals described in the above section. Finally, these intervals for each analyte were implemented in the LIS.

\section{Post-implementation prospective study}

One year after implementing the system, between September 2018 and April 2019 (eight months), a study was conducted to establish the numbers of requests in the emergency and routine laboratories in each of the four analyte intervals. The percentages were compared with those obtained in the pre-implementation study for the same intervals. Pre- and post-implementation results were obtained from the Omnium database (Oracle; Roche Diagnostics).

\section{Data analysis}

Data analysis, interferogram generation, and calculation of straightline trend value sand $R^{2}$ regression coefficients were carried out in spreadsheets (Excel, Microsoft, Redmond, WA, USA).

\section{RESULTS}

According to the representation model proposed by Glick, et al. [15] (Fig. 1), LDH showed the highest degree of positive hemolysis interference, which reached $50 \%$ at a $\mathrm{Hb}$ concentration of approximately $1 \mathrm{~g} / \mathrm{L}$. DB reached $50 \%$ of interference (negative) at a $\mathrm{Hb}$ concentration of $0.5-1 \mathrm{~g} / \mathrm{L}$. AST and folic acid reached $50 \%$ interference at $3 \mathrm{~g} / \mathrm{L}$, but above that concentration, folic acid caused a higher percentage of interference than AST. Potassium was the only analyte selected that did not reach the required interference level until a $\mathrm{Hb}$ concentration of $10 \mathrm{~g} / \mathrm{L}$. How- ever, it was included in the study for comparison with the other analytes as it is a classical hemolysis marker [20].

Cut-offs were established using the BV levels published in the SEQC ${ }^{\mathrm{ML}}$ and EFLM BV databases $\left(\mathrm{CV}_{\mathrm{I}}\right)$ and the laboratory $\mathrm{CV}_{\mathrm{A}}$ level (Table 1). For two analytes, DB and folic acid, CV levels are present only in the SEQC ${ }^{\mathrm{ML}}$ database, whereas for the other three analytes, $\mathrm{CV}_{\text {I }}$ levels are present in both databases. The cut-offs for potassium were the most restrictive, whereas those for folic acid and DB were the most permissive.

For all analytes except DB, the regression line was linear, with $\mathrm{R}^{2}>0.98$. For $\mathrm{DB}$, the regression curve was exponential, with $\mathrm{R}^{2}>0.98$ (Fig. 2) [16]. In all five interferograms, the regression line crossed the axes at coordinates $(0,0)$.

Table 2 lists the concentrations of the intervals established for the management of hemolysis. $\mathrm{LDH}$ and DB presented low values in intervals 1 and 2. AST, potassium, and folic acid were associated with a higher $\mathrm{Hb}$ concentration at the same level of interference. Results of the various analytes above the concentrations in interval 2 were not reported to clinicians. This trend was observed for both emergency and routine samples.

In a pre-implementation study using emergency and routine samples (Table 3), a low percentage of LDH levels were reported correctly in the routine laboratory (28.16\% of total requests); $71.84 \%$ of the cases were, but should not have been reported given the interval defined in Table 2.

As for routine $\mathrm{LDH}$, the percentage of samples in intervals 2 $(2.52 \%)$ and $3(0.21 \%)$ decreased between the pre- and postimplementation stages, and that of samples in interval 1 increased $(2.72 \%)$ (Table 4). This trend was generally not observed in the emergency samples. The percentages for DB were obtained using a small number of hemolyzed samples because we only an-

Table 1. CV and $\mathrm{CV}_{\mathrm{A}}$ levels used to calculate analytical and clinical cut-offs

\begin{tabular}{lccccccc}
\hline Analyte & $\begin{array}{c}\text { CV } \\
\text { SEQC }\end{array}$ & $\begin{array}{c}\text { CVL } \\
\text { EFLM }\end{array}$ & CV $_{\text {A }}$ & $\begin{array}{c}\text { Analytical cut-off } \\
\text { SEQC }\end{array}$ & $\begin{array}{c}\text { Clinical cut-off } \\
\text { SEQC }\end{array}$ & $\begin{array}{c}\text { Analytical cut-off } \\
\text { EFLM DQS }\end{array}$ & $\begin{array}{c}\text { Clinical cut-off } \\
\text { EFLM RCV }\end{array}$ \\
\hline AST & 12.3 & 9.5 & 3.6 & 6.54 & 35.5 & 5.6 & 28.1 \\
DB $^{*}$ & 36.8 & - & 7.2 & 14.19 & 103.9 & - & - \\
Potassium & 4.6 & 4.1 & 1.3 & 1.81 & 13.3 & 1.5 & 11.9 \\
LDH & 8.6 & 5.2 & 3.8 & 4.26 & 26.1 & 3.4 & 17.9 \\
Folic acid* & 24 & - & 8.2 & 19.21 & 70.3 & - & - \\
\hline
\end{tabular}

Data are expressed as percentages.

Analytical cut-off: value of the DQS described in the BV database of the SEQC ${ }^{\mathrm{ML}}$ and the EFLM for each column.

Clinical cut-off: value of the RCV calculated from the equation described in the Materials and Methods section from SEQC ${ }^{\mathrm{ML}}$ and the EFLM data for each column.

${ }^{*} \mathrm{CV}_{1}$ and $\mathrm{DQS}$ levels were extracted from the BV database of the SEQC ${ }^{\mathrm{ML}}$ alone because they were not available in the BV database of the EFLM. Abbreviations: AST, aspartate aminotransferase; $\mathrm{CV}_{1}$, within-subject biological variation; $\mathrm{CV}_{\mathrm{A}}$, analytical variation; $\mathrm{DB}$, direct bilirubin; $\mathrm{LDH}$, lactate dehydrogenase; EFLM, European Federation of Laboratory Medicine; RCV, reference change value; BV, biological variation; DQS, desirable quality specification; SEQC ${ }^{\mathrm{ML}}$, Spanish Society of Laboratory Medicine. 
A

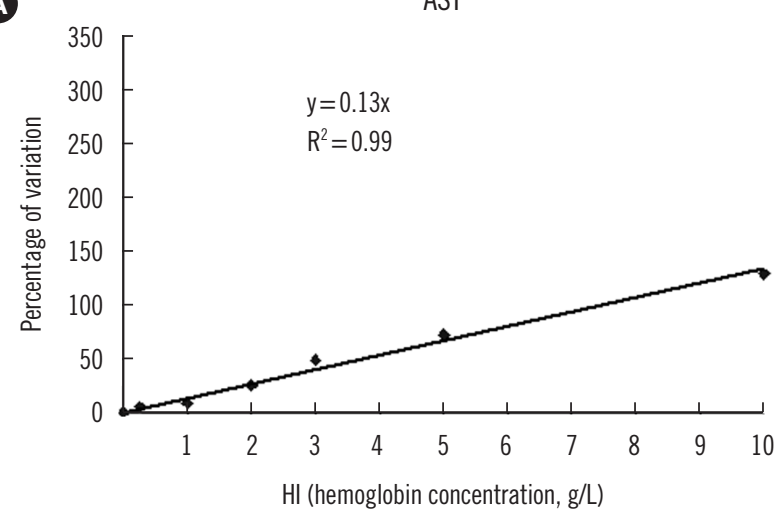

c

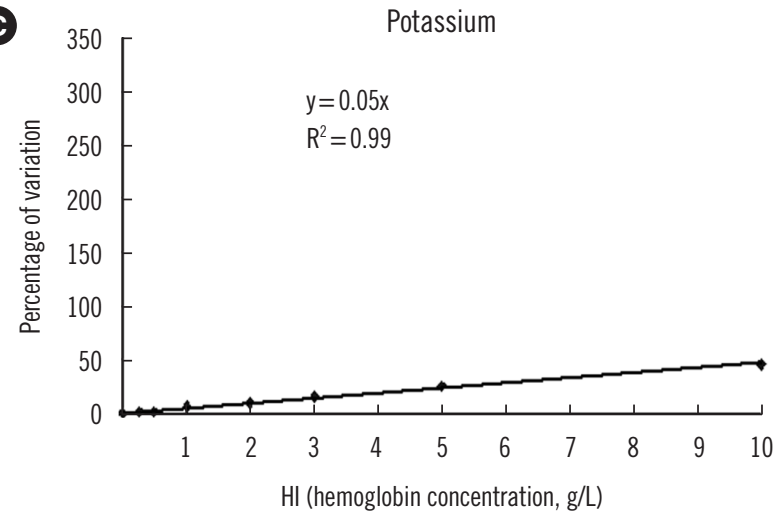

$\boldsymbol{B}$

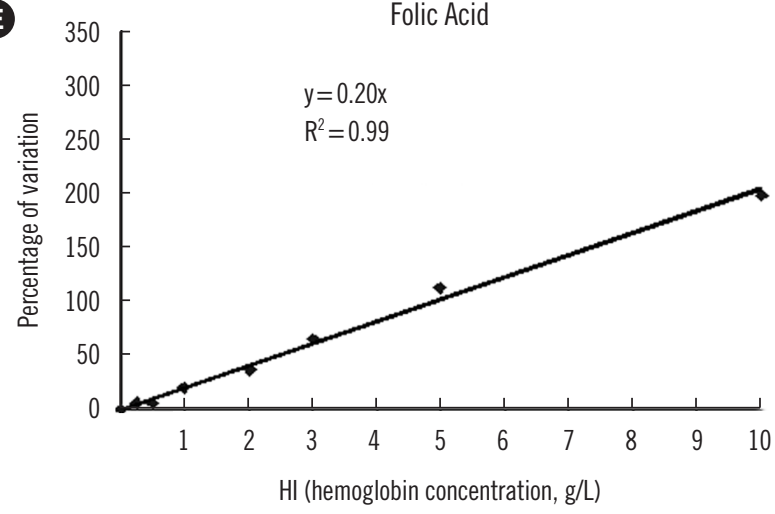

alyzed whether the total bilirubin concentration was above the reference interval; therefore, the results could not be assessed.

\section{DISCUSSION}

Currently, there is no consensus on the criteria to establish cutoffs for hemolysis interference. The CLSI-EP07-A2 guidelines [21] suggest variation $>10 \%$ as a significant cut-off for hemolysis interference, regardless of the analyte being studied. The Working Group on Laboratory Errors and Patient Safety of the International Federation of Clinical Chemistry has developed
B

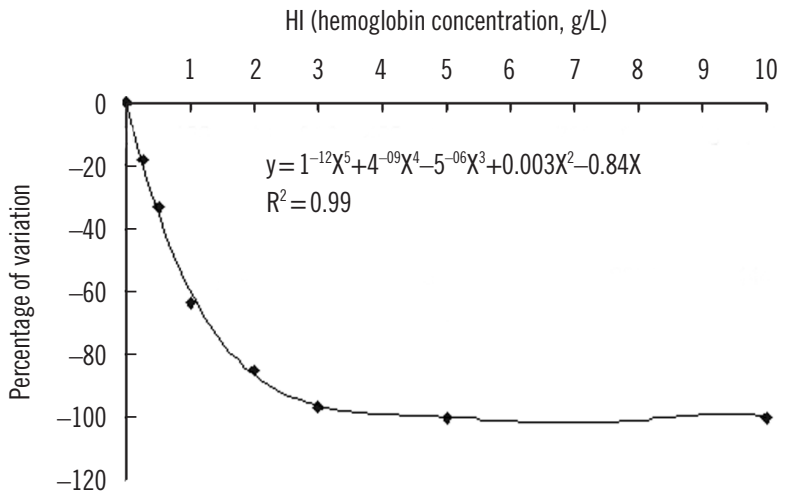

D

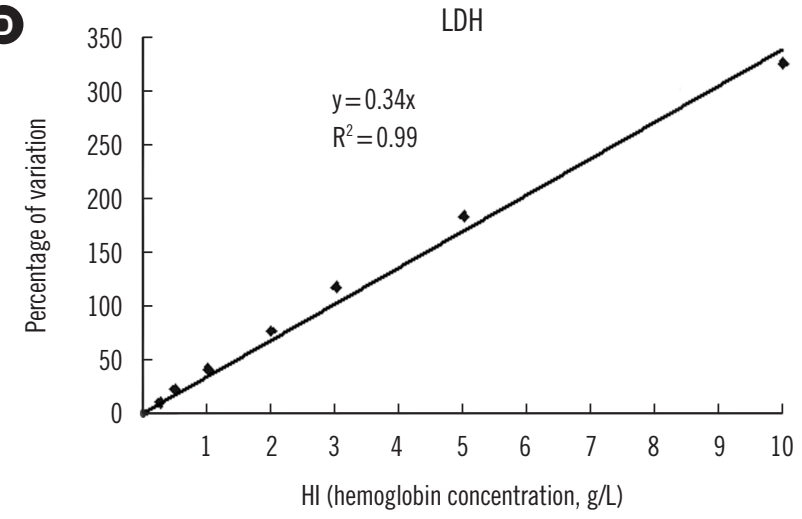

Fig. 2. The regression line is linear for all analytes except $D B$, for which it is exponential. Best-fit regression lines are shown. The regression line equation and regression coefficient value are shown in the figure.

Abbreviations: AST, aspartate aminotransferase; DB, direct bilirubin; $\mathrm{LDH}$, lactate dehydrogenase; $\mathrm{HI}$, hemolysis index.

quality indicators for comparison between different clinical laboratories [22]. Among preanalytical indicators, the indicator of hemolyzed samples was defined as the number of samples with a free $\mathrm{Hb}$ concentration $>0.5 \mathrm{~g} / \mathrm{L}$ divided by the total number of samples analyzed in the laboratory [23]. Finally, in vitro diagnostics (IVD) companies generally suggest a cut-off of $10 \%$ for hemolysis interference, regardless of the analyte being studied. We established cut-offs for five selected analytes and a working algorithm and acceptance and rejection criteria for the results based on the interference level. Following the recommendations of the EFLM WG-PRE [13], four intervals were established based 
Table 2. Cut-offs established for pre- and post-implementation studies

\begin{tabular}{lcccc}
\hline \multirow{2}{*}{ Analyte } & \multicolumn{4}{c}{ Interval (g/L) } \\
\cline { 2 - 5 } & Interval 1 & Interval 2 & Interval 3 & Interval 4 \\
\hline AST & $0-0.46$ & $0.46-2.65$ & $2.65-10$ & $\geq 10$ \\
DB & $0-0.22$ & $0.22-0.70$ & $0.70-10$ & $\geq 10$ \\
Potassium & $0-0.09$ & $0.09-2.65$ & $2.65-10$ & $\geq 10$ \\
LDH & $0-0.11$ & $0.11-0.56$ & $0.56-10$ & $\geq 10$ \\
Folic acid & $0-0.92$ & $0.92-3.85$ & $3.85-10$ & $\geq 10$
\end{tabular}

Data represent the concentration of free $\mathrm{Hb}$ in $\mathrm{g} / \mathrm{L}$. The intervals were calculated from the DQS and RCV values based on the BV data of the SEQC ${ }^{\mathrm{ML}}$. Interval 1: below the DQS value, interval 2: between DQS and RCV values, interval 3: above the RCV value, and interval 4: $\mathrm{HI}>10 \mathrm{~g} / \mathrm{L}$.

Abbreviations: AST, aspartate aminotransferase; DB, direct bilirubin; $L D H$, lactate dehydrogenase; DQS, desirable quality specification; RCV, reference change value; $\mathrm{BV}$, biological variation. on BV criteria (DQS and RCV), and they were implemented in the LIS and standardized with comments on the report.

The intervals were calculated from BV data in the SEQC ${ }^{\mathrm{ML}}$ database [18] because EFLM BV data [19] were not available at the time of the study (2017-2018). However, we observed no significant differences regarding the management of hemolyzed samples comparing the values obtained for both databases (SE$\mathrm{QC}^{\mathrm{ML}}$ and EFLM BV) in the pre- and post-implementation studies (data not shown). The use of BV data from the EFLM BV database is recommended for subsequent studies because they are more robust.

Based on our data, the number of analytes with interference $>10 \%$ at a free $\mathrm{Hb}$ concentration of $0.5 \mathrm{~g} / \mathrm{L}$ was very low (data not shown). An increase in the cut-off up to an interference of

Table 3. Pre-implementation study of the percentages of samples for which analytical results were reported and not reported for each of the selected analytes

\begin{tabular}{|c|c|c|c|c|c|c|}
\hline \multirow[b]{2}{*}{ Analyte } & \multicolumn{3}{|c|}{ Emergency } & \multicolumn{3}{|c|}{ Routine } \\
\hline & $\begin{array}{l}\text { Should have } \\
\text { been reported }\end{array}$ & $\begin{array}{c}\text { Should not have } \\
\text { been reported }\end{array}$ & $\begin{array}{l}\text { Adequately } \\
\text { reported }\end{array}$ & $\begin{array}{l}\text { Should have } \\
\text { been reported }\end{array}$ & $\begin{array}{c}\text { Should not have } \\
\text { been reported }\end{array}$ & $\begin{array}{c}\text { Adequately } \\
\text { reported }\end{array}$ \\
\hline$\overline{\text { AST }(2.65 \mathrm{~g} / \mathrm{L})^{\dagger}}$ & 0.45 & 9.06 & 90.94 & 0.07 * & $60.78^{*}$ & 39.22 \\
\hline $\mathrm{DB}(0.7 \mathrm{~g} / \mathrm{L})^{\dagger}$ & $0.05^{*}$ & $65.28 *$ & 34.72 & 0 * & $72.22 *$ & 27.78 \\
\hline Potassium $(2.64 \mathrm{~g} / \mathrm{L})^{\dagger}$ & 0.54 & 7.48 & 92.52 & 0.26 & 4.89 & 95.11 \\
\hline $\mathrm{LDH}(0.56 \mathrm{~g} / \mathrm{L})^{\dagger}$ & 0.65 & 73.62 & 23.38 & 0.02 & 71.84 & 28.16 \\
\hline Folic acid $(3.85 \mathrm{~g} / \mathrm{L})^{\dagger}$ & - & - & - & $0.15^{*}$ & $50^{*}$ & 50 \\
\hline
\end{tabular}

Data represent the percentages of adequately reported analytes, those that should have been reported, and those that should not have been reported considering the cut-off for hemolysis established between intervals 2 and 3 (shown in brackets for each analyte). An analyte was classified as adequately reported when it was reported with a hemolysis level below the cut-off shown in parentheses, and it was classified as inadequately reported when it was reported despite exceeding the established cut-off.

*Values for which the number of results is $<100$; ${ }^{\dagger}$ Free $\mathrm{Hb}$ concentration $(\mathrm{g} / \mathrm{L})$.

Abbreviations: AST, aspartate aminotransferase; DB, direct bilirubin; LDH, lactate dehydrogenase.

Table 4. Percentages of pre- and post-implementation requests for the five analytes in emergency and routine laboratories

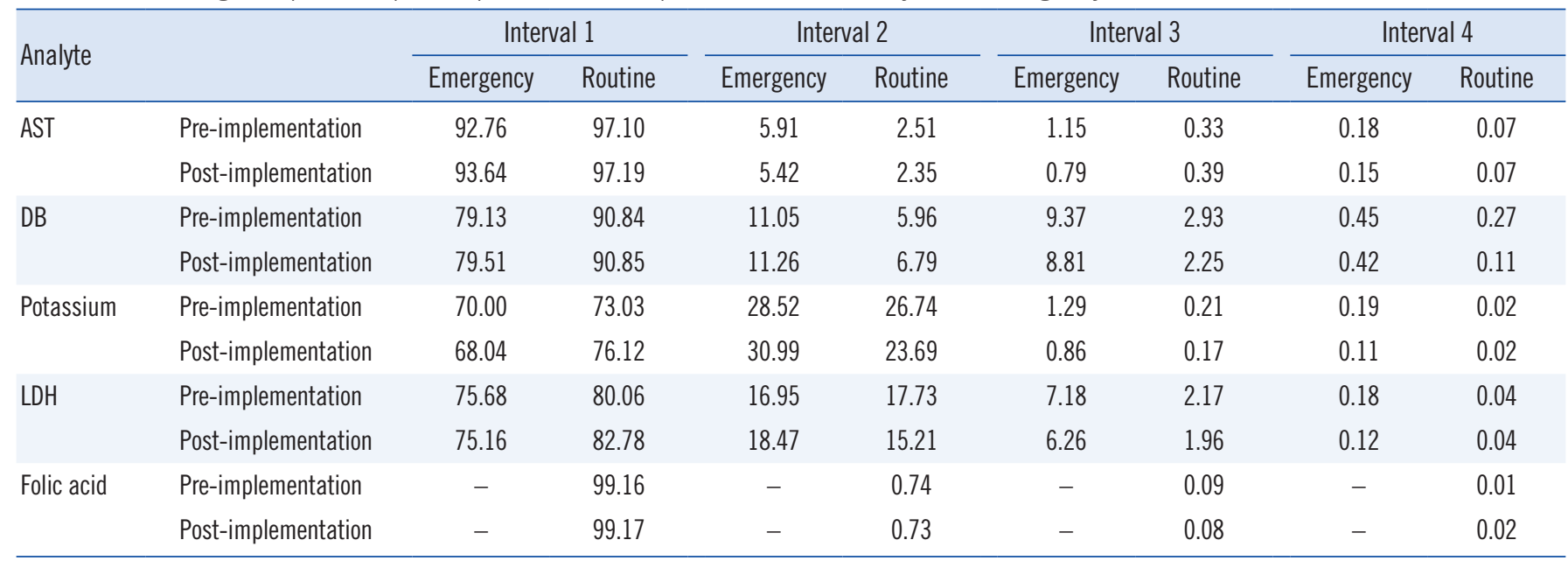

Abbreviations: AST, aspartate aminotransferase; $\mathrm{DB}$, direct bilirubin; $\mathrm{LDH}$, lactate dehydrogenase. 
$50 \%$ at a $\mathrm{Hb}$ concentration $>5 \mathrm{~g} / \mathrm{L}$ allowed us to select the anaIytes with the highest levels of interference to which the protocol for hemolysis management was applied, i.e., LDH, AST, DB, potassium, and folic acid. Given their interference levels, these analytes provided more information to assess the study objectives than others.

Analyte-by-analyte hemolysis management yields individual degrees of interference and provides more reliable information to practitioners, helping them with result interpretation. Therefore, based on the interferograms, we observed that for four analytes (all except DB), the analytical results were overestimated. The difference in their behavior is associated with their interference mechanisms: LDH, AST, potassium, and folic acid are released from RBCs, whereas decreased concentrations of DB are due to chemical interference [6]. Therefore, it is essential that clinical laboratories establish their own cut-offs for adequate hemolysis management.

Gils, et al. [11] established an $\mathrm{HI}$ reference value of $0.16 \mathrm{~g} / \mathrm{L}$ in a healthy population using the same analytical platform we used. In our study, in interval 1, potassium and LDH showed maximum concentrations of 0.09 and $0.11 \mathrm{~g} / \mathrm{L}$, respectively, which is below the limit of $0.16 \mathrm{~g} / \mathrm{L}$ proposed by Gils, et al. [11]. Therefore, our protocol reduces the undervaluation of hemolysis compared with the use of a fixed reference limit for all analytes in a sample. Conversely, AST, DB, and folic acid had cut-offs $>0.16 \mathrm{~g} / \mathrm{L}$ in interval 1 , indicating that a fixed limit for the entire sample would overestimate hemolysis. Therefore, this protocol allows a more individualized interference management and increases patient safety by reducing erroneous interpretation of analytical results. The use of cut-offs based on BV allows us to be more restrictive in those analytes with narrower $\mathrm{CV}_{\text {, levels, }}$ such as potassium, in which concentrations $>0.09 \mathrm{~g} / \mathrm{L}$ are overestimated based on interval 1 in our protocol versus $2 \mathrm{~g} / \mathrm{L}$ with a variation of $10 \%$ (CLSI criterion) [21]. Before this study was conducted, in our laboratory, we determined hemolysis based on the cut-off established by the IVD provider $(0.15 \mathrm{~g} / \mathrm{L}$ for any analyte) and generally discarded the results of analytes, such as potassium or $\mathrm{LDH}$, without including informative comments.

To prove the efficacy of the algorithm that was implemented in the LIS, pre- and post-implementation studies were conducted to analyze its impact on the management of hemolyzed samples. The pre-implementation study used the upper limit of interval 2 as a cut-off for each analyte to observe how the samples would have been managed. For potassium, the percentage of samples that were adequately reported in the routine laboratory based on this upper limit would have been higher than that in the emer- gency laboratory (95.11\% vs. 92.52\%). A similar behavior was observed for incorrectly reported samples (4.89\% vs. $7.48 \%$ ), based on the new levels in our study. The percentage of correctly reported samples for LDH was significantly low in both routine and emergency laboratories (28.16\% vs. $26.38 \%$ ), resulting in a high percentage of incorrectly reported samples ( $71.84 \%$ vs. $73.62 \%)$. LDH is more sensitive to hemolysis than other anaIytes, whereas potassium is sensitive to inadequate sample transport. $\mathrm{LDH}$ is released from RBC only when the membrane breaks (hemolysis), whereas potassium is transported through the intact membrane, which may account for the differences between these two analytes [24].

The comparison of the results at the pre- and post-implementation stages showed an improvement in the percentage of hemolyzed samples in the routine laboratory, with a decreased percentage of samples in interval 2 and an increased percentage in interval 1 , mainly for $\mathrm{LDH}$ and potassium. The protocol allowed us to increase the percentage of reported samples without hemolysis interference (interval 1). However, the post-implementation stage was not studied in the emergency laboratory, and an opposite trend was observed regarding the percentages of samples in intervals 1 and 2 . In both the emergency and the routine analytes, a slight decrease in hemolysis levels was found in intervals 3 and 4; however, the results are not significant considering the low number of samples in these two groups.

The implementation of a harmonized protocol for hemolysis management makes it possible to achieve homogeneous criteria for reporting by clinical laboratories. Moreover, informing the requesting practitioners about the degree and type of interference (under- or overestimation) with pre-established comments leads to a better clinical interpretation of the results. We have observed a progressive improvement of the quality of the samples received in the laboratory (lower degree of hemolysis), probably due to a better understanding of the clinical impact of interference by the requesting practitioners.

Individual laboratories should establish the degree of hemolysis interference for all analytes, which is affected by the analytical methodology and the equipment used. The establishment of BV-based individualized cut-offs for interference management makes it possible to implement a harmonized protocol for hemolysis management in the laboratory. Moreover, the implementation of such a protocol would make it possible to compare results across laboratories that assess hemolysis based on individual analytes. This protocol is endorsed by recommendations, such as those of the EFLM WG-PRE [13]. In our laboratory, there was an improvement in terms of adequate detection of the in- 
terference level caused by hemolysis for each analyte and a decrease in the percentage of samples affected by interference after the protocol had been implemented and assessed. A periodical assessment of results would help in the establishment of an internal monitoring protocol focused on the preanalytical stage and serving as an internal control tool. More studies of this kind using other analytical platforms (with different method-instrument relationships) will be needed to assess their effectiveness. Finally, the implementation of this protocol has a high impact on the quality of the results, allowing better clinical decisions and increasing patient safety.

\section{ACKNOWLEDGEMENTS}

None.

\section{AUTHOR CONTRIBUTIONS}

Fernando Marques-Garcia has participated in the study design and the acquisition, analysis, and interpretation of the data.

David Hansoe Heredero Jung has participated in the study design and the acquisition, analysis, and interpretation of the data.

Sandra Elena Pérez has participated in the study design and the acquisition, analysis, and interpretation of the data.

All authors revised the paper and approved of the version to be published. All authors agreed to be accountable for all aspects of the work in ensuring that questions related to the accuracy or integrity of any part of the work are appropriately investigated and resolved.

\section{CONFLICTS OF INTEREST}

None declared.

\section{RESEARCH FUNDING}

None declared.

\section{ORCID}

Fernando Marques-Garcia

https://orcid.org/0000-0002-2324-4003

David Hansoe Heredero Jung

https://orcid.org/0000-0002-6631-9319

Sandra Elena Pérez

https://orcid.org/0000-0002-3825-1142

\section{REFERENCES}

1. Goyal T and Schmotzer CL. Validation of hemolysis index thresholds optimizes detection of clinically significant hemolysis. Am J Clin Patho 2015;143:579-83.

2. Lippi G, Blanckaert N, Bonini P, Green S, Kitchen S, Palicka V, et al. Haemolysis: an overview of the leading cause of unsuitable specimens in clinical laboratories. Clin Chem Lab Med 2008;46:764-72.

3. Plebani M. The detection and prevention of errors in laboratory medicine. Ann Clin Biochem 2010;47:101-10.

4. Plebani M and Lippi G. Closing the brain-to-brain loop in laboratory testing. Clin Chem Lab Med 2011;49:1131-3.

5. Farrell CJ and Carter AC. Serum indices: managing assay interference. Ann Clin Biochem 2016;53:527-38.

6. Marqués-García F. Methods for hemolysis interference study in laboratory medicine - a critical review. EJIFCC 2020;31:85-97.

7. Minović I, Dikkeschei LD, Vos MJ, Kootstra-Ros JE. Interpretation of folate results in hemolytic plasma samples: a practical approach. Ann Lab Med. 2021;41:485-8.

8. Howanitz PJ, Lehman CM, Jones BA, Meier FA, Horowitz GL. Clinical laboratory quality practices when hemolysis occurs. Arch Pathol Lab Med 2015;139:901-6

9. Luksic AH, Nikolac Gabaj N, Miler M, Dukic L, Bakliza A, Simundic AM. Visual assessment of hemolysis affects patient safety. Clin Chem Lab Med 2018;56:574-81.

10. Dupuy AM, Bargnoux AS, Kuster N, Cristol JP, Badiou S. Determination of hemolysis cut-offs for biochemical and immunochemical analytes according to their value. Clin Chem Lab Med 2020;58:1232-41.

11. Gils C, Sandberg MB, Nybo M. Verification of the hemolysis index measurement: imprecision, accuracy, measuring range, reference interval and impact of implementing analytically and clinically derived sample rejection criteria. Scand J Clin Lab Invest 2020;80:580-9.

12. CLSI. Haemolysis, Icterus, and Lipemia/Turbidity Indices as Indicators of interference in Clinical Laboratory Analysis: Approved Guideline. 1st ed., CLSI C56-A. Wayne, PA: Clinical and Laboratory Standards Institute. 2012

13. Lippi G, Cadamuro J, Von Meyer A, Simundic AM, European Federation of Clinical Chemistry and Laboratory Medicine (EFLM) Working Group for Preanalytical Phase (WG-PRE). Practical recommendations for managing hemolyzed samples in clinical chemistry testing. Clin Chem Lab Med 2018;56:718-27.

14. Meites S. Letter: Reproducibly simulating hemolysis, for evaluating its interference with chemical methods. Clin Chem 1973;19:1319.

15. Glick MR, Ryder KW, Jackson SA. Graphical comparisons of interferences in clinical chemistry instrumentation. Clin Chem 1986;32:470-5.

16. Pum JKW. Evaluating sample stability in the clinical laboratory with the help of linear and non-linear regression analysis. Clin Chem Lab Med 2020;58:188-96.

17. Aarsand AK, Røraas T, Bartlett WA, Coşkun A, Carobene A, FernandezCalle $P$, et al. Harmonization initiatives in the generation, reporting and application of biological variation data. Clin Chem Lab Med 2018;56: 1629-36.

18. Minchinela J, Ricós $C$, Perich $C$, Fernández-Calle P, Álvarez V, Doménech MV, et al. Biological variation database and quality specifications for imprecision, bias and total error (desirable and minimum). The 2014 update. https://www.westgard.com/biodatabase-2014-update.htm (Updated on Nov 2020).

19. Aarsand AK, Fernandez-Calle P, Webster C, Coskun A, Gonzales-Lao E, Diaz-Garzon J, et al. The EFLM Biological Variation Database. https://bi- 
Marques-Garcia $\mathrm{F}$, et al.

Hemolysis management based on biological variation

ologicalvariation.eu/ (Updated on Feb 2021).

20. Wan Azman WN, Omar J, Koon TS, Tuan Ismail TS. Hemolyzed specimens: major challenge for identifying and rejecting specimens in clinical laboratories. Oman Med J 2019;34:94-8.

21. CLSI. Interference testing in clinical chemistry. 3rd ed. EP07-02. Wayne, PA: Clinical and Laboratory Standards Institute. 2021.

22. Plebani M, Sciacovelli L, Aita A, Pelloso M, Chiozza ML. Corrigendum to: Performance criteria and quality indicators for the pre-analytical phase. Clin Chem Lab Med 2015;53:1653.

23. Sciacovelli L, Lippi G, Sumarac Z, West J, Garcia Del Pino Castro I, Furtado Vieira K, et al. Quality indicators in laboratory medicine: the status of the progress of IFCC working group "Laboratory Errors and Patient Safety" project. Clin Chem Lab Med 2017;55:348-57.

24. Zaninotto M, Tasinato A, Padoan A, Vecchiato G, Pinato A, Sciacovelli L, et al. Effects of sample transportation on commonly requested laboratory tests. Clin Chem Lab Med 2012;50:1755-60. 